\title{
Neuroscience-Informed Cognitive-Affective Training Interventions for Mood and Anxiety Disorders
}

Evan Alvarez, M.A. ${ }^{1, *}$ Brian M. Iacoviello, Ph.D. ${ }^{2}$

\author{
Address \\ ${ }^{*}, 1$ New York State Psychiatric Institute, Columbia University, 1051 Riverside Drive, \\ Suite 2501, New York, NY 10032, USA \\ Email: ealvare@nyspi.columbia.edu \\ ${ }^{2}$ Department of Psychiatry, Icahn School of Medicine at Mount Sinai, 1 Gustave L \\ levy PlaceBox 1230, New York, NY 10029, USA
}

Published online: 8 July 2015

(C) Springer International Publishing AG 2015

This article is part of the Topical Collection on Mood Disorders

Keywords Mood · Anxiety · Cognitive $\cdot$ Affective $\cdot$ Training $\cdot$ Intervention

\section{Opinion statement}

Mood and anxiety disorders are characterized by abnormally persistent affective states. Neuroimaging studies have implicated the functioning of specific brain regions in the aberrant affective processing observed in mood and anxiety disorders: enhanced engagement of subcortical regions responsible for affect generation (amygdala, hippocampus) is coupled with reduced inhibitory cognitive control of affective processing from dorsolateral and ventrolateral prefrontal cortices. Emerging but compelling evidence suggests that neuroplastic mechanisms can be harnessed to remediate these abnormalities, thus offering the prospect of new therapeutic interventions. Cognitive-affective training is a particularly promising strategy for enhancing cognitive control over affective processes via modulation of these abnormally functioning neural networks. This paper presents a review of the current literature and a justification for developing cognitive-affective training, and proposes initial evidence for the efficacy of cognitive-affective training interventions. Cognitive-affective training interventions could represent a novel method for targeting perseverative thinking and negative affective biases in mood and anxiety disorders. 


\section{Introduction}

Mood disorders and anxiety disorders are characterized by the abnormal persistence of affective symptoms and cognitive abnormalities [1-4] that contribute to poor psychosocial functioning and outcome in these patient populations. Mood and anxiety disorders have consistently been identified as a leading cause of overall disease burden worldwide because of their significant public health cost, morbidity, and mortality [5]. This is partially due to the lack of highly effective treatments. Despite numerous pharmacological agents available, approximately $30-40 \%$ of patients do not achieve adequate therapeutic response [6-8].

Several psychologically based interventions have also been developed. Cognitive behavioral therapy (CBT) [9-12] and interpersonal psychotherapy (IPT) [13, 14] are two commonly utilized, evidence-based psychotherapies for treating anxiety and depression. Although promising post-treatment and follow-up remission rates have been observed in numerous randomized trials [12, $14,15]$, studies investigating long-term treatment outcomes have reported notable decreases in remission rates over time [16-18], with anxiety and depressive symptoms often returning to clinically significant levels. Failure to achieve sustained rates of remission in mood and anxiety disorders has prompted the development of novel, alternative interventions designed to target the key underlying deficits in neural network functioning.

Cognitive bias modification (CBM) represents a first attempt at a neuroscience-based intervention. CBM directly targets pathological cognitive content (e.g., negative interpretations of events) or cognitive processes (e.g., biased orienting of immediate attention toward negatively valenced stimuli) in mood and anxiety disorders [19]. Despite initially promising studies [20], meta-analyses of the preliminary research findings on CBM interventions suggest that treatment effects are generally small to non-significant in anxious and depressed samples [21•], especially when administered in routine clinical settings. Nonetheless, CBM has been very useful in introducing the concept of neuroscienceinformed cognitive-affective training interventions that can be further refined to improve short- and long-term outcomes.

It is generally acknowledged that the efficacy gap observed in treatment reflects misalignment between the mode of action of the intervention and the mechanisms underlying disease expression. The need to address this gap has been clearly articulated as a major strategic goal of the National Institute of Mental Health (NIMH) that mandates "research that translates emerging findings on the neuroscience and behavioral science of mental disorders into novel psychosocial (e.g., cognitive strategies and innovative behavioral approaches) and other non-pharmacological interventions...that will alter dysfunctional neural circuits and psychological processes underlying mental disorders to reduce symptoms" [22].

The opportunities for the development of novel translational interventions continue to grow as we build upon our understanding of the intersection between neuroscience, cognitive science, and psychiatry. In this paper, we describe the rationale and method for developing cognitive-affective training interventions for patients suffering from mood and anxiety disorders and provide an example of how this could be applied in the treatment of major depressive disorder (MDD).

\section{Relevant neuroscience findings informing the development of cognitive-affective training for mood and anxiety disorders}

The basic premise of a neuroscience-informed strategy for cognitive-affective training is that repeated training targets psychopathology (particularly cognitive and affective processing biases) by way of enhancing top-down cognitive control and modulates neural network dysfunction through brain plasticity mechanisms to effect remedial change $[23,24]$. The neural networks underlying affect processing have been explored in healthy individuals, and abnormalities in these networks have been consistently shown in patients with disorders. The overlap in neural abnormalities among these disorders highlights the 
opportunity for applying trans-diagnostic cognitive-affective training interventions that target these mechanisms as potential new intervention strategies.

Neural networks involved in affect processing

Upon perception, affective information is relayed to the thalamus, which projects to the amygdala/hippocampal complex [25]. The amygdala and hippocampus, proximal structures that exhibit mutual facilitation during this processing, evaluate the affective salience of the stimuli $[26,27]$. The ventral striatum (also referred to as the nucleus accumbens) is another region, intricately connected to the amygdala/hippocampus complex and projecting to the thalamus, which appears to preferentially promote processing of rewarding or positive stimuli [28]. Additional bottom-up information is also processed by the subgenual cingulate cortex (SGC) [29]. Input from these regions is mostly directed to the medial prefrontal cortex (mPFC), a region involved in conscious awareness of the affective salience of information and in self-referential processing [30-33]. Top-down cognitive control, on the other hand, is primarily exerted by lateral, ventral, and dorsal prefrontal cortical (PFC) regions, particularly the dorsolateral PFC (dlPFC) and dorsal anterior cingulate cortex (dACC) [26, 27]. In healthy individuals, amygdala activity increases during the processing of affective information [27] and shows an inverse relationship with dlPFC activity [26, 34-36].

An expansive magnetic resonance imaging (MRI) literature has contributed to a detailed delineation of the brain regions implicated in mood and anxiety disorders, both in terms of structure (volume) and function.

Several meta-analyses have summarized the relevant literature in mood disorders [particularly MDD and bipolar disorder (BD)] and, to a lesser extent, in anxiety disorders. In terms of MDD, structural MRI (sMRI) studies have shown that the most consistent findings are volumetric decreases in the anterior cingulate cortex (ACC) extending further upwards to the ventromedial PFC (vmPFC), as well as the dlPFC, and downwards to the amygdala, hippocampus, and parts of the striatum (particularly the putamen) [37-40]. In BD, there are now several reviews and meta-analyses that have synthesized the extensive primary sMRI literature [38, 41-45]. Consistent volumetric reductions between patients compared to healthy individuals have been observed in the vmPFC, amygdala, hippocampus, insula, and ACC [42, 44, 45]. In anxiety disorders, the literature is more limited. Nevertheless, volumetric reductions compared to healthy controls have been reliably identified in the amygdala, hippocampus, and the vmPFC in patients with post-traumatic stress disorder (PTSD) [46-48], panic disorder (PD) $[49,50]$, generalized anxiety disorder (GAD), and phobias [51].

The functioning (activity) of the regions implicated in affect processing, and inter-regional interactions, has also been examined using functional MRI (fMRI) both during task-related and task-independent ("resting state") conditions. Regardless of condition, individuals with MDD demonstrate hyperactivity notably in the thalamus, the amygdala/hippocampal complex, and the ventral striatum [52-55]. In contrast, concurrent hypoactivity has been observed in lateral PFC structures, particularly during the processing of affective information [56]; this is believed to contribute to the impaired cognitive control and perseverative thinking symptoms observed. Similarly in patients with BD, hyperactivation is observed in the amygdala/hippocampus complex, the putamen, and the thalamus, with hypoactivation in lateral PFC regions [54]. A 
similar pattern of increased limbic hyperactivation with prefrontal underengagement has been reported across anxiety disorders [55-57].

Taken together, the evidence presented above suggests that persistent affective dysregulation has a diagnosis-independent neural signature that is characterized by structural and functional abnormalities within the neural circuits involved in affect processing. Specifically, reduced lateral PFC-related cognitive control over medial cortical and subcortical regions involved in affect generation and processing result in biased and prolonged processing of negative affective information (e.g., perseverative worrying, ruminating, etc.). Figure 1 presents a schematic depiction of the neural network dysfunction underlying the abnormal cognitive-affective processing observed in mood and anxiety disorders and representing targets for cognitive-affective training interventions.

\section{Neuroplasticity}

Neuroplasticity refers to the brain's ability to change due to experience and alter future responses to related stimuli [58]. Neuroplasticity is a multifaceted phenomenon that can involve functional changes such as the strengthening or weakening of connections between neurons and remodeling of synapses [59, 60] or structural changes such as neurogenesis. Synaptic remodeling is a core dimension of neuroplasticity. Synaptic pruning and synaptogenesis refer to the idea that neuronal connections are routinely being removed or recreated based upon how they are experientially used [61•]. A fundamental principle is that frequent neuronal co-activation leads to the creation of new synapses or the fortifying of

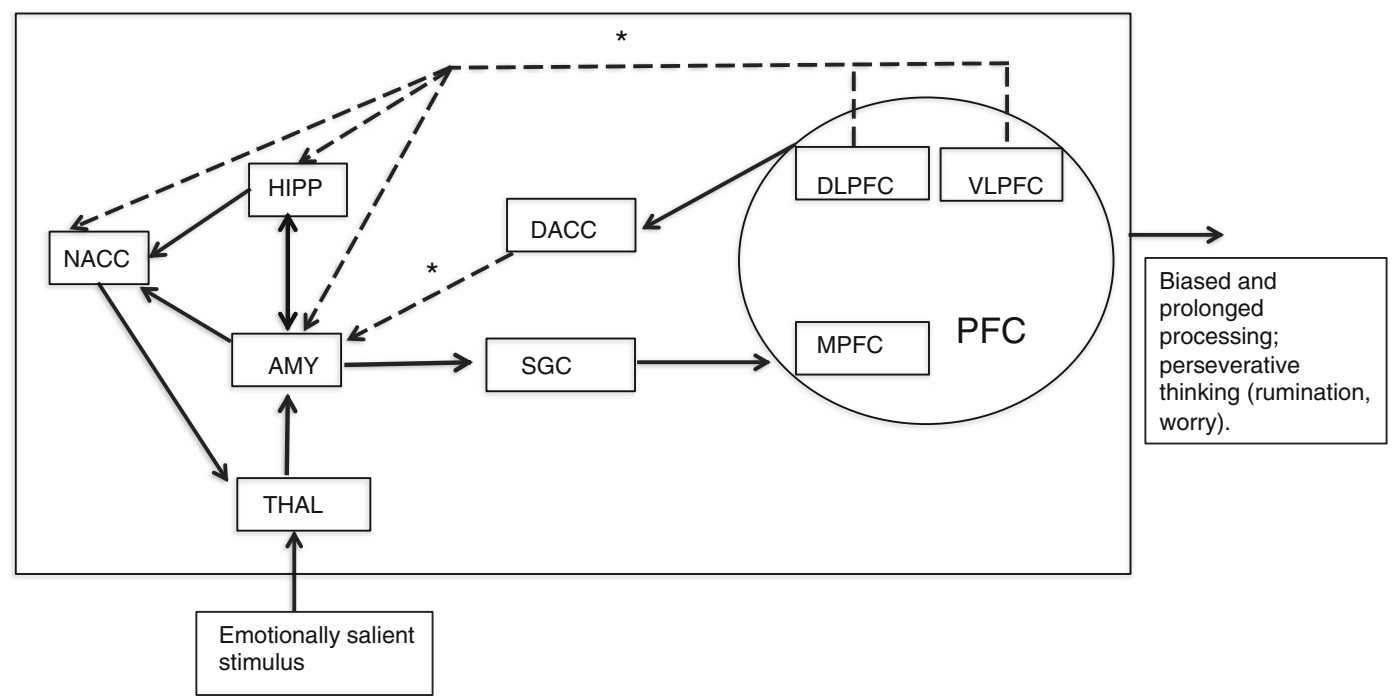

Fig. 1. A neurobiological model for cognitive-affective processing abnormalities in mood and anxiety disorders and targets for interventions. Hyperactivation of the thalamus (THAL), amygdala (AMY), and hippocampus (HIPP) upon perception of emotionally salient stimuli is associated with increased subgenual cingulate cortex (SGC) activity. SGC activity is associated with increased medial PFC (mPFC) activity; this activity integrates limbic feedback and relays further upstream to other prefrontal cortex (PFC) regions. Concurrently, PFC regions (dlPFC, vlPFC) and the dorsal anterior cingulate cortex (dACC)-which facilitate inhibitory control over limbic regions and are associated with cognitive control and emotion regulation-are observed to be hypoactive. The functional connectivity between the PFC and other brain regions (AMY, HIPP) appears weakened, which suggests poor cognitive control over these regions. Dashed lines and asterisks indicate functional connectivity abnormalities that result in impaired cognitive control and emotion regulation, and delineate targets for cognitive-affective training interventions. 
existing ones. Alternatively, the synaptic connections between neurons that do not regularly co-activate weaken. Consequently, the quantity and strength of brain synapses can change over time, impacting neural network functioning.

Whole cell plasticity, also known as homeostatic plasticity, is the change in the excitability of neurons [62]. Neuronal excitability reflects the assimilation of excitatory postsynaptic potentials (EPSPs) and inhibitory postsynaptic potentials (IPSPs) and is influenced by the function of ion channels in the axon, dendrites, and cell body. Changes to the electrical properties of neurons can influence spike generation, synaptic integration, and other core mechanisms at the cellular level. Higher order brain functioning, such as learning and memory in particular, can be altered as a result of these individual neuronal changes.

Neurogenesis may also contribute to neuroplasticity. However, in the adult brain, this contribution is likely to be less widespread compared to synaptic changes and homeostatic plasticity. In the adult brain, neurogenesis is mostly convincingly demonstrated in the hippocampus $[63,64]$ and in the subventricular zone (SVZ) [65]. More recent evidence implicates further brain regions not commonly identified as sites of neurogenesis, including the neocortex $[66,67]$. Taken collectively, synaptic pruning, synaptogenesis, homeostatic plasticity, and, to a lesser extent, neurogenesis begin to explicate mechanisms by which brain plasticity can unfold on structural and functional levels.

Preclinical and clinical studies provide preliminary support for the notion that cognitive interventions can influence neuroplasticity. Preclinical studies, for example, using a neonatal ventral hippocampal lesion rat model of schizophrenia, have demonstrated that cognitive training in adolescent rats conferred protection against cognitive control deficits and modified hippocampal neural activity as a mechanism of effect [68].

Clinical studies using PET and fMRI involving human subjects receiving cognitive therapy for depression and anxiety identified functional changes in neural circuits responsible for cognitive control and affect, specifically by increasing dlPFC activity and subsequently reducing limbic system activity during affective information processing [69]. Studies have also shown that cognitive priming for emotional stimuli had a persistent effect on subsequent amygdala activity [70]. A systems neuroscience rationale has also been proposed for the possible mechanisms of cognitive-affective training interventions. This model emphasizes the distributed infrastructure of neural circuits that maintain cog-

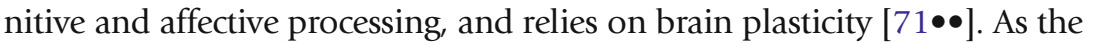
human brain learns, cognitive/affective inputs and action outputs ultimately become manifested by greater and more coordinated groups of neurons across multiple brain regions and levels of processing. Taken together, cognitively based interventions that include cognitive-affective training may have the potential to effect change in brain neurobiology and functioning in ways that are consistent with improvements in clinical outcomes.

\section{Neuroscience-informed cognitive-affective training: paradigm and proof of concept application in MDD}

We have developed a new cognitive-affective training intervention for MDD. The intervention aims to enhance cognitive control of emotional information 
processing by specifically targeting abnormal activation patterns in dIPFC and amygdala that underlie the cognitive control and emotion regulation impairments observed in MDD. To accomplish this, the intervention combined working memory and facial affect identification tasks, which have been shown to specifically target activity in the dlPFC [72] and amygdala [73], respectively. The task performed during the intervention requires that patients identify the emotions expressed on a series of faces displayed on a computer screen while remembering the specific sequence of faces. The working memory component of the task utilizes an N-back working memory paradigm in which participants indicate if the emotion expressed on a currently viewed face matches with the emotion $\mathrm{N}$ faces back. Depending on the patient's performance, the $\mathrm{N}$ level varies; $\mathrm{N}$ can increase or decrease across all blocks while patients are instructed to complete many blocks for each session. Administering the task in this way effectively calibrates to the participants' ability level while proactively challenging them as a means of enhancing engagement of the targeted brain structures. The target engagement of this task has been validated using fMRI techniques in a single, non-progressive session in healthy volunteers; it simultaneously recruits dIPFC structures involved in working memory and limbic structures involved in affective processing (amygdala) [74].

Proof of concept for the efficacy of this intervention in MDD has been provided in a pilot study [75•]. The interventions are based on the assumption that exercising the ability to manipulate emotional information in working memory could enhance cognitive control for emotional information processing via modulation of the corresponding neural networks. Enhancement of cognitive control is expected to reduce rumination and depressive symptoms. Accordingly, we conducted a placebo-controlled clinical trial of our cognitive-affective training intervention. Twenty-one patients with MDD participated in this trial and were seen twice per week for 4 weeks. Half the sample received the experimental task while the other half was randomly assigned to an active sham task, which involved a straightforward working memory exercise using an adaptive N-back task with shapes as the stimuli, not emotional faces. This task is known to activate the dlPFC but not the amygdala. The effectiveness of the cognitive-affective training was evaluated using a battery of measures to assess: (1) changes in cognitive biases and rumination; (2) changes in neurocognition/working memory; and (3) changes in MDD symptoms. At study outcome, significant differences in reductions in negative affective bias in short-term memory and MDD symptoms were reported between the groups [75•] but both groups showed similar, small improvements in working memory. These findings ultimately suggest that cognitive-affective training holds the promise of enhancing cognitive control for affective information processing and modulating the underlying neural networks, and that these changes could have antidepressant or antianxiety effects by fortifying emotion regulation and reducing the perseverative thinking (e.g., worrying, rumination) observed in mood and anxiety disorders.

\section{Conclusion}

As the body of knowledge about the connection between cognitive and affective neuroscience and psychopathology continues to grow, opportunities to develop and refine novel intervention strategies capitalizing on brain plasticity will 
grow with it. Empirical support is currently the strongest for strategies like cognitive bias modification and cognitive therapies. The development of cognitive training exercises as neurobehavioral therapies, which target underlying neural components linked to the pathophysiology of specific disorders, is now also a possibility. The intention of this paper is to provide an outline for how researchers can develop, and clinicians employ, cognitive-affective training interventions to treat anxiety and mood disorders in non-research settings. Further research is still needed for understanding the full effects of these mechanisms. Finally, subsequent studies may also investigate the effects of cognitive-affective interventions as an augmentation to current evidence-based psychotherapy or pharmacotherapy treatments for anxiety and mood disorders, as well as the sustainability of training effects after the regimen is completed.

\section{Compliance with Ethics Guidelines}

\section{Conflict of Interest}

Evan Alvarez declares that he has no conflict of interest. Brian M. Iacoviello reports grants from the National Institute of Mental Health and grants from Brain and Behavior Research Foundation (issuing NARSAD grants) during the conduct of the study.

\section{Human and Animal Rights and Informed Consent}

This article includes review of studies conducted by one or more authors of the current paper, in which human subjects participated, but does not present new or unpublished data from these studies.

\section{References and Recommended Reading}

Papers of particular interest, published recently, have been highlighted as:

- Of importance

$\bullet \quad$ Of major importance

1. Stefanopoulou E, Manoharan A, Landau S, Geddes JR, Goodwin G, Frangou S. Cognitive functioning in patients with affective disorders and schizophrenia: a meta-analysis. Int Rev Psychiatry. 2009;21(4):336-56.

2. McIntyre RS, Cha DS, Soczynska JK, Woldeyohannes HO, Gallaugher LA, Kudlow P. Cognitive deficits and functional outcomes in major depressive disorder: determinants, substrates, and treatment interventions. Depress Anxiety. 2013;30(6):515-27.

3. Bora E, Harrison BJ, Yücel M, Pantelis C. Cognitive impairment in euthymic major depressive disorder: a meta-analysis. Psychol Med. 2013;43(10):2017-26.

4. Depp CA, Mausbach BT, Harmell AL, Savla GN, Bowie CR, Harvey PD, et al. Meta-analysis of the association between cognitive abilities and everyday functioning in bipolar disorder. Bipolar Disord. 2012;14(3):217-26.

5. Whiteford HA, Degenhardt L, Rehm J, Baxter AJ, Ferrari AJ, Erskine HE, et al. Global burden of disease attributable to mental and substance use disorders: findings from the Global Burden of Disease Study 2010. Lancet. 2013;382(9904):1575-86.

6. Magalhães PV, Dodd S, Nierenberg AA, Berk M. Cumulative morbidity and prognostic staging of illness in the Systematic Treatment Enhancement Program for Bipolar Disorder (STEP-BD). Aust N Z J Psychiatry. 2012. doi:10.1177/0004867412460593.

7. Bandelow B, Baldwin DS, Dolberg OT, Andersen HF, Stein DJ. What is the threshold for symptomatic response and remission for major depressive disorder, panic disorder, social anxiety disorder, and generalized anxiety disorder? J Clin Psychiatry. 2006;67(9):1428-34.

8. Offidani E, Guidi J, Tomba E, Fava GA. Efficacy and tolerability of benzodiazepines versus antidepressants in anxiety disorders: a systematic review and metaanalysis. Psychother Psychosom. 2013;82(6):355-62.

9. Beck JS. Cognitive behavior therapy: basics and beyond. Guilford Press. 2011. 
10. Piacentini J, Bennett S, Compton SN, Kendall PC, Birmaher B, Albano AM, et al. 24-and 36-week outcomes for the Child/Adolescent Anxiety Multimodal Study (CAMS). J Am Acad Child Adolesc Psychiatry. 2014;53(3):297-310.

11. March J, Silva S, Petrycki S, Curry J, Wells K, Fairbank J, et al. Fluoxetine, cognitive-behavioral therapy, and their combination for adolescents with depression: Treatment for Adolescents With Depression Study (TADS) randomized controlled trial. JAMA: J Am Med Assoc. 2004;292(7):807-20.

12. Hofmann SG, Smits JA. Cognitive-behavioral therapy for adult anxiety disorders: a meta-analysis of randomized placebo-controlled trials. J Clin Psychiatry. 2008;69(4):621.

13. Klerman GL, Weissman MM. Interpersonal psychotherapy of depression: a brief, focused, specific strategy. Jason Aronson, Incorporated. 1994.

14. de Mello MF, de Jesus Mari J, Bacaltchuk J, Verdeli H, Neugebauer R. A systematic review of research findings on the efficacy of interpersonal therapy for depressive disorders. Eur Arch Psychiatry Clin Neurosci. 2005;255(2):75-82.

15. Reinecke MA, Ryan NE, Dubois DL. Cognitivebehavioral therapy of depression and depressive symptoms during adolescence: a review and metaanalysis. J Am Acad Child Adolesc Psychiatry. 1998;37(1):26-34.

16. Norton PJ, Price EC. A meta-analytic review of adult cognitive-behavioral treatment outcome across the anxiety disorders. J Nerv Ment Dis. 2007;195(6):521-31.

17. Ginsburg GS, Becker EM, Keeton CP, Sakolsky D, Piacentini J, Albano AM, et al. Naturalistic follow-up of youths treated for pediatric anxiety disorders. JAMA Psychiatry. 2014;71(3):310-8.

18. Ravitz P, McBride C, Maunder R. Failures in interpersonal psychotherapy (IPT): factors related to treatment resistances. J Clin Psychol. 2011;67(11):1129-39.

19. Bar-Haim Y. Research review: attention bias modification (ABM): a novel treatment for anxiety disorders. J Child Psychol Psychiatry. 2010;51(8):859-70.

20. Hakamata Y, Lissek S, Bar-Haim Y, Britton JC, Fox NA Leibenluft E, et al. Attention bias modification treatment: a meta-analysis toward the establishment of novel treatment for anxiety. Biol Psychiatry. 2010;68(11):982-90.

21. Cristea IA, Kok RN, Cuijpers P. Efficacy of cognitive bias modification interventions in anxiety and depression: meta-analysis. Br J Psychiatry. 2015;206(1):7-16.

This review paper summarizes the cognitive training approaches developed and studied to date, focusing on cognitive bias modification exercises, but not neuroscience-informed cognitive-affective training interventions.

22. National Institutes of Health. Translational research for the development of novel interventions for mental disorders. 2011. Available from: http://www.nimh.nih. gov/research-funding/grants/concept-clearances/2011/ translational-research-for-the-development-of-novelinterventions-for-mental-disorders.shtml.
23. Iacoviello BM, Charney DS. Developing cognitiveemotional training exercises as interventions for mood and anxiety disorders. Eur Psychiatry. 2015;30(1):75-81.

24. Siegle GJ, Ghinassi F, Thase M. Neurobehavioral therapies in the 21st century: summary of an emerging field and an extended example of cognitive control training for depression. Cogn Ther Res. 2007;31(2):235-62.

25. LeDoux J. The emotional brain: the mysterious underpinnings of emotional life. Simon and Schuster. 1998.

26. Davidson RJ. Affective style, psychopathology, and resilience: brain mechanisms and plasticity. Am Psychol. 2000;55(11):1196.

27. Drevets WC. Neuroimaging and neuropathological studies of depression: implications for the cognitiveemotional features of mood disorders. Curr Opin Neurobiol. 2001;11(2):240-9.

28. Nestler EJ, Carlezon WA. The mesolimbic dopamine reward circuit in depression. Biol Psychiatry. 2006;59(12):1151-9.

29. Greicius MD, Flores BH, Menon V, Glover GH, Solvason HB, Kenna H, et al. Resting-state functional connectivity in major depression: abnormally increased contributions from subgenual cingulate cortex and thalamus. Biol Psychiatry. 2007;62(5):429-37.

30. Gusnard DA, Akbudak E, Shulman GL, Raichle ME. Medial prefrontal cortex and self-referential mental activity: relation to a default mode of brain function. Proc Natl Acad Sci. 2001;98(7):4259-64.

31. Fossati P, Hevenor SJ, Graham SJ, Grady C, Keightley ML, Craik F, et al. In search of the emotional self: an fMRI study using positive and negative emotional words. Am J Psychiatr. 2003;160(11):1938-45.

32. Denson TF, Pedersen WC, Ronquillo J, Nandy AS. The angry brain: neural correlates of anger, angry rumination, and aggressive personality. J Cogn Neurosci. 2009;21(4):734-44.

33. Ray RD, Ochsner KN, Cooper JC, Robertson ER, Gabrieli JD, Gross JJ. Individual differences in trait rumination and the neural systems supporting cognitive reappraisal. Cogn Affect Behav Neurosci. 2005;5(2):156-68.

34. Fales CL, Barch DM, Rundle MM, Mintun MA, Snyder AZ, Cohen JD, et al. Altered emotional interference processing in affective and cognitive-control brain circuitry in major depression. Biol Psychiatry. 2008;63(4):377-84.

35. Costafreda SG, Brammer MJ, David AS, Fu CH. Predictors of amygdala activation during the processing of emotional stimuli: a meta-analysis of 385 PET and fMRI studies. Brain Res Rev. 2008;58(1):57-70.

36. Siegle GJ, Steinhauer SR, Thase ME, Stenger VA, Carter CS. Can't shake that feeling: event-related fMRI assessment of sustained amygdala activity in response to emotional information in depressed individuals. Biol Psychiatry. 2002;51(9):693-707.

37. Sacher J, Neumann J, Fünfstück T, Soliman A, Villringer A, Schroeter ML. Mapping the depressed brain: a metaanalysis of structural and functional alterations in 
major depressive disorder. J Affect Disord. 2012;140(2):142-8.

38. Kempton MJ, Salvador Z, Munafo MR, Geddes JR, Simmons A, Frangou S, et al. Structural neuroimaging studies in major depressive disorder: meta-analysis and comparison with bipolar disorder. Arch Gen Psychiatry. 2011;68(7):675-90.

39. Koolschijn PCMP, van Haren NE, Lensvelt-Mulders GJ, Pol H, Hilleke E, Kahn RS. Brain volume abnormalities in major depressive disorder: a meta-analysis of magnetic resonance imaging studies. Hum Brain Mapp. 2009;30(11):3719-35.

40. Cole J, Costafreda SG, McGuffin P, Fu CH. Hippocampal atrophy in first episode depression: a metaanalysis of magnetic resonance imaging studies. J Affect Disord. 2011;134(1):483-7.

41. Hallahan B, Newell J, Soares JC, Brambilla P, Strakowski SM, Fleck DE, et al. Structural magnetic resonance imaging in bipolar disorder: an international collaborative mega-analysis of individual adult patient data. Biol Psychiatry. 2011;69(4):326-35.

42. Ellison-Wright I, Bullmore E. Anatomy of bipolar disorder and schizophrenia: a meta-analysis. Schizophr Res. 2010;117(1):1-12.

43. Vita A, De Peri L, Sacchetti E. Gray matter, white matter, brain, and intracranial volumes in first-episode bipolar disorder: a meta-analysis of magnetic resonance imaging studies. Bipolar Disord. 2009;11(8):807-14.

44. Bora E, Pantelis C. Structural trait markers of bipolar disorder: disruption of white matter integrity and localized gray matter abnormalities in anterior frontolimbic regions. Biol Psychiatry. 2011;69(4):299-300.

45. Selvaraj S, Arnone D, Job D, Stanfield A, Farrow TF, Nugent AC, et al. Grey matter differences in bipolar disorder: a meta-analysis of voxel-based morphometry studies. Bipolar Disord. 2012;14(2):135-45.

46. Karl A, Schaefer M, Malta LS, Dörfel D, Rohleder N, Werner A. A meta-analysis of structural brain abnormalities in PTSD. Neurosci Biobehav Rev. 2006;30(7):1004-31.

47. Woon FL, Sood S, Hedges DW. Hippocampal volume deficits associated with exposure to psychological trauma and posttraumatic stress disorder in adults: a meta-analysis. Prog Neuro-Psychopharmacol Biol Psychiatry. 2010;34(7):1181-8.

48. Kühn S, Gallinat J. Gray matter correlates of posttraumatic stress disorder: a quantitative meta-analysis. Biol Psychiatry. 2013;73(1):70-4.

49. Sobanski T, Wagner G, Peikert G, Gruhn U, Schluttig K, Sauer $\mathrm{H}$, et al. Temporal and right frontal lobe alterations in panic disorder: a quantitative volumetric and voxel-based morphometric MRI study. Psychol Med. 2010;40(11):1879-86.

50. Lai CH, Wu YT. Fronto-temporo-insula gray matter alterations of first-episode, drug-naive and very lateonset panic disorder patients. J Affect Disord.

2012;140(3):285-91.

51. Shang J, Fu Y, Ren Z, Zhang T, Du M, Gong Q, et al. The common traits of the ACC and PFC in anxiety disorders in the DSM-5: meta-analysis of voxel-based morphometry studies. PLoS One. 2014;9(3):e93432.

52. Price JL, Drevets WC. Neurocircuitry of mood disorders. Neuropsychopharmacology. 2010;35(1):192-216.

53. Iwabuchi SJ, Krishnadas R, Li C, Auer D, Radua J, Palaniyappan L. Localized connectivity in depression: a meta-analysis of resting state functional imaging studies. Neuroscience \& Biobehavioral Reviews. 2015.

54. Delvecchio G, Fossati P, Boyer P, Brambilla P, Falkai P, Gruber $\mathrm{O}$, et al. Common and distinct neural correlates of emotional processing in bipolar disorder and major depressive disorder: a voxel-based meta-analysis of functional magnetic resonance imaging studies. Eur Neuropsychopharmacol. 2012;22(2):100-13.

55. Fredrikson M, Faria V. Neuroimaging in anxiety disorders. Mod Trends Pharmacopsychiatry. 2012;29:47-66.

56. Ramage AE, Laird AR, Eickhoff SB, Acheson A, Peterson AL, Williamson DE, et al. A coordinate-based metaanalytic model of trauma processing in posttraumatic stress disorder. Hum Brain Mapp. 2013;34(12):3392-9.

57. Hattingh CJ, Ipser J, Tromp SA, Syal S, Lochner C, Brooks SJ, et al. Functional magnetic resonance imaging during emotion recognition in social anxiety disorder: an activation likelihood meta-analysis. Front Hum Neurosci. 2012;6:1.

58. Pascual-Leone A, Amedi A, Fregni F, Merabet LB. The plastic human brain cortex. Annu Rev Neurosci. 2005;28:377-401.

59. Cramer SC, Sur M, Dobkin BH, O'Brien C, Sanger TD, Trojanowski JQ, et al. Harnessing neuroplasticity for clinical applications. Brain. 2011;134(6):1591-609.

60. Calabrese F, Molteni R, Racagni G, Riva MA. Neuronal plasticity: a link between stress and mood disorders. Psychoneuroendocrinology. 2009;34:S208-16.

61. Kourrich S, Bonci A. Synaptic and neuronal plasticity. In: Charney DS, Buxbaum JD, Sklar P, Nestler EJ, editors. Neurobiology of mental illness. Oxford University Press. 2013. This chapter outlines the mechanisms by which neural plasticity occurs and can be harnessed for cognitive-affective training purposes.

62. Eriksson PS, Perfilieva E, Björk-Eriksson T, Alborn AM, Nordborg C, Peterson DA, et al. Neurogenesis in the adult human hippocampus. Nat Med. 1998;4(11):1313-7.

63. Gould E, Reeves AJ, Fallah M, Tanapat P, Gross CG, Fuchs E. Hippocampal neurogenesis in adult Old World primates. Proc Natl Acad Sci. 1999;96(9):5263-7.

64. Gould E, Reeves AJ, Graziano MS, Gross CG. Neurogenesis in the neocortex of adult primates. Science. 1999;286(5439):548-52.

65. Lim DA, Alvarez-Buylla A. Interaction between astrocytes and adult subventricular zone precursors stimulates neurogenesis. Proc Natl Acad Sci. 1999;96(13):7526-31.

66. Zhao M, Momma S, Delfani K, Carlén M, Cassidy RM, Johansson CB, et al. Evidence for neurogenesis in the 
adult mammalian substantia nigra. Proc Natl Acad Sci. 2003;100(13):7925-30.

67. Shankle WR, Rafii MS, Landing BH, Fallon JH. Approximate doubling of numbers of neurons in postnatal human cerebral cortex and in 35 specific cytoarchitectural areas from birth to 72 months. Pediatr Dev Pathol. 1999;2(3):244-59.

68. Lee H, Dvorak D, Kao HY, Duffy ÁM, Scharfman HE, Fenton AA. Early cognitive experience prevents adult deficits in a neurodevelopmental schizophrenia model. Neuron. 2012;75(4):714-24.

69. DeRubeis RJ, Siegle GJ, Hollon SD. Cognitive therapy versus medication for depression: treatment outcomes and neural mechanisms. Nat Rev Neurosci. 2008;9(10):788-96.

70. Pichon S, Rieger SW, Vuilleumier P. Persistent affective biases in human amygdala response following implicit priming with negative emotion concepts. NeuroImage. 2012;62(3):1610-21.

71.• Vinogradov S, Fisher M, de Villers-Sidani E. Cognitive training for impaired neural systems in neuropsychiatric illness. Neuropsychopharmacology.

2012;37(1):43-76.
This paper summarizes the relevant literature on the neurobiological rational and systems involved in cognitive training interventions.

72. Dima D, Jogia J, Frangou S. Dynamic causal modeling of load-dependent modulation of effective connectivity within the verbal working memory network. Hum Brain Mapp. 2014;35(7):3025-35.

73. Dima D, Stephan KE, Roiser JP, Friston KJ, Frangou S. Effective connectivity during processing of facial affect: evidence for multiple parallel pathways. J Neurosci. 2011;31(40):14378-85.

74. Neta $M$, Whalen PJ. Individual differences in neural activity during a facial expression vs. identity working memory task. Neuroimage. 2011;56(3):1685-92.

75. Iacoviello BM, Wu G, Alvarez E, Huryk K, Collins KA, Murrough JW, et al. Cognitive-emotional training as an intervention for major depressive disorder. Depress Anxiety. 2014;31(8):699-706.

This research report provides preliminary evidence for the efficacy of a neuroscience-informed cognitive-affective training intervention for MDD. 